\title{
Crystallography open database for teaching
}

Saulius Grazulis ${ }^{1}$, Andrius Merkys ${ }^{1}$, Antanas Vaitkus ${ }^{1}$

${ }^{1}$ Vilnius University Institute Of Biotechnology, Vilnius, Lithuania

E-mail: grazulis@ibt.It

In the today's swiftly changing world, it is especially important that students get access to comprehensive volumes of up to date information. Developing skills for making inferences from the data as well as handling data itself is mandatory for modern curriculum. Open databases play crucial part in this process, since they permit both an unlimited re-use and sharing of data in the classroom as well as participation in real-life data gathering and curation tasks.

The Crystallography Open Database (COD, [1]) provides an excellent tool for such teaching approach. After an initial introduction into crystallographic data handling, motivated students get individual assignments for data analysis and curation of the Crystallography Open Database. While working on these problems, students learn in practice how to perform various tasks using live crystallographic data. Using COD allows to present students with real-life, up to date problems. Initial solutions are checked by a supervisor, and if found good are committed to the COD under mutual agreement with students. As students gain experience, they receive subsequently more challenging tasks. After a period of initial training, the most motivated students can perform studies on their own, and use the COD as a research tool for their bachelor, MSc and PhD theses. The skills gained during initial assignments allow students to quickly join the research team, participate in projects and co-author joint publications [2-3]. For students, such arrangement gives rich work experience and early participation in research; the open nature of the COD allows students to continue their work using the same tools even if they later decide to leave the group. For the group, a large benefit arises from the skilled work force that permits to participate in various national and international projects. For example, the current COD team recruited some former MSc and current PhD students to participate in three calls from the Research Council of Lithuania ( 2 were granted financing), and in the currently running SOLSA project from the European Union under the Horizon 2020 program.

The report will reveal several personal success stories, emphasize strengths of this approach; we will also discuss challenges that we met while implementing our approach and expose what could be considered as weaknesses of our current teaching method.

1. The Crystallography Open Database Web site (2017), http://www.crystallography.net/ [accessed 2017-02-27].

2. Gražulis, S. et al. (2009). Crystallography Open Database - an open-access collection of crystal structures, J. Appl. Crystallogr. 42, 726-729.

3. Gražulis, S. et al. (2012). Crystallography Open Database (COD): an open-access collection of crystal structures and platform for world-wide collaboration, Nucleic Acids Res. 40, D420-D427.

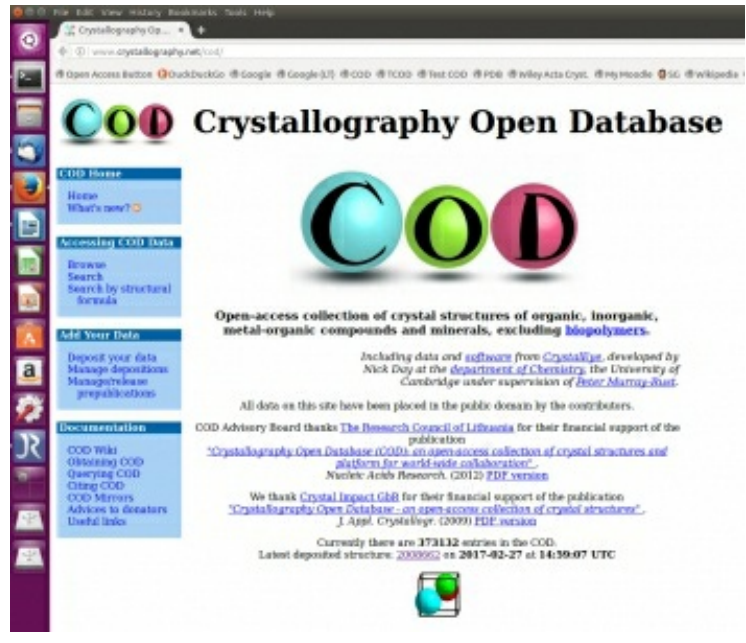

Keywords: Crystallography Open Database, hands-on learning 